\title{
MATERIAL AND COMPONENT VALIDATION BY SPECKLE INTERFEROMETRY AND CORRELATION METHODS
}

\section{A.Ettemeyer}

Ettemeyer Consulting, Ulm, Germany

\begin{abstract}
Challenging the limits of modern material and component designs requires a deep understanding of the product performance with respect to stress distribution, fatigue and lifetime. With the help of modern simulation tools as FEA this process has significantly been improved and simplified. However, for experimental validation in the test, modern and efficient test methods have to be provided.
\end{abstract}

Here, the speckle technique offers an excellent potential. Electronic speckle pattern interferometry (ESPI) has been proven to be a flexible and efficient tool for characterization of material properties of complex materials. Inversing the measuring principle by moving the illumination instead of the object, the ESPI technique also enables contour measurement functions, which have been integrated into the ESPI technique. This enlarged the application range to the inspection of components under load and for direct comparison of the measured contour and strain data with the FEA simulation.

In order to meet the increasing need for the analysis of a larger range of deformations, e.g. in forming processes or for inspection of polymers, the development of a new digital 3D image correlation technique was initiated. Two cameras observe the object under investigation and calculate the $3 \mathrm{D}$ surface of the component using photogrammetric algorithm. The correlation of images, taken at different load levels leads to the complete information about the deformations and strains on each point of the surface.

In this paper, we compare both techniques and give examples of successful applications.

Introduction: Modern materials and components are often loaded far beyond the former strain limits. Especially in automotive and aircraft industries, they have to satisfy the toughest strength requirements and meet stringent safety standards. At the same time, product cycles have dramatically been shortened. To reduce cost and time-to-market, rapid design optimisation has become a key issue.

In the last decade, many industries adopted Computer-Aided-Engineering (CAE) tools to reduce the number of prototype builds and to speed up the development cycle. These analytical tools are relatively inexpensive to use and faster to implement than the costly traditional test methods as used in traditional design processes. There are, however, many variables that CAE tools cannot adequately address, such as, boundary conditions, material anisotropy etc. Therefore, efficient physical experimental techniques are still necessary for component design validation.

A traditional experimental technique for determining component strain/stress characteristics is the application of strain gauges. Although inexpensive and effective for some applications, strain gauges provide only localized information and cannot capture true peak strain or high-resolution information when applied in regions having large strain gradient. Strain gauges also require time consuming and careful application, which have also limited its utility for design optimisation in an intensive, time-critical development effort.

Modern optical measuring techniques based on Speckle Interferometry and on Digital Image Correlation Techniques have opened new opportunities for fast and effective investigations on materials and components behaviour.

Electronic Speckle Pattern Interferometry: Electronic Speckle Pattern Interferometry (ESPI) is a full field non-contact experimental measuring technique that allows rapid and highly accurate measurement of the three components of deformation and generation of strain/stress distribution with high resolution, /1/. It is well suited for the determination of the true peak strain and the strain distribution in regions of strain concentration that are usually the most critical in an investigation. Fig. 1 shows a schematic of an ESPI set-up. The laser beam is split into two beams, one as the object illumination beam and the other as a reference beam. The light reflected from the object surface is recombined with the reference beam. This results in an interference pattern that is the socalled speckle pattern and is recorded by the CCD camera. Before the object is deformed, the phase difference between the object and the reference beams is $\phi$. After the object is deformed, the phase difference becomes $(\phi$ $+\Delta)$ and a phase change $\Delta$ results from the deformation. The subtraction of both phase distributions $\phi$ and $(\phi+$ 
$\Delta$ ) gives the phase change $\Delta$ which is related to an object deformation in the direction of the sensitivity vector $\mathbf{S}$ /2/. As an example a clamped circular plate loaded centrally is presented in Fig. 1

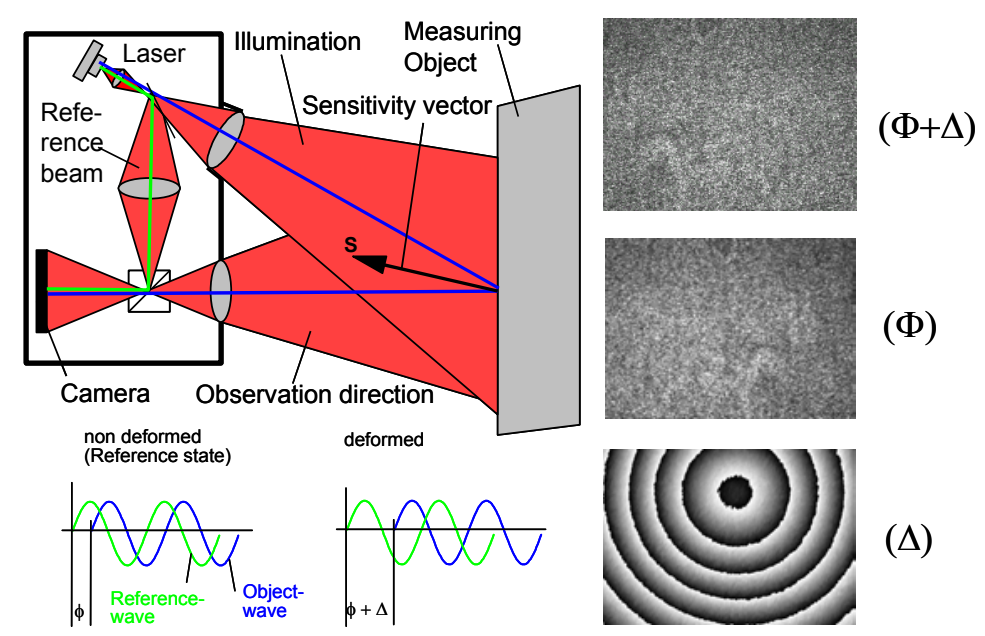

Fig. 1: Principle of ESPI technique

For measurement of the complete 3D displacement of the object, the measurement is repeated with minimum 3 different laser illumination directions enabling the measurement of the same object deformation with 3 different sensitivity vectors $S_{1}, S_{2}, S_{3}$. For practical reasons, generally 4 symmetric illuminations are used, Fig. 2, and the laser illumination is automatically switched 4 times each at the acquisition of the reference state and the deformed state. The complete image acquisition is performed within typ. 1 second. The measurement resolution for displacements is a fraction of the laser wavelength and typ. $30 \ldots 100 \mathrm{~nm}$.

Principle of Strain Measurement: As described above, 3D-ESPI enables rapid and accurate determination of the three components of deformation $\mathrm{u}, \mathrm{v}$ and $\mathrm{w}$ on an objects surface. Because it is of a full field measurement, the distributions of $\mathrm{u}, \mathrm{v}$ and $\mathrm{w}$ over the entire object surface i.e. the functions $\mathrm{u}(\mathrm{x}, \mathrm{y}), \mathrm{v}(\mathrm{x}, \mathrm{y})$ and $\mathrm{w}(\mathrm{x}, \mathrm{y})$ can be obtained, $/ 3 /$.

Obviously, the in-plane strains at each point of the measured area can be determined by differentiating the in-plane components $\mathrm{u}(\mathrm{x}, \mathrm{y})$ and $\mathrm{v}(\mathrm{x}, \mathrm{y})$ :

$$
\varepsilon_{x}=\frac{\partial u}{\partial x}, \quad \varepsilon_{y}=\frac{\partial v}{\partial y}, \quad \gamma_{x y}=\frac{\partial u}{\partial y}+\frac{\partial v}{\partial x}
$$

Such differentiation can be carried out, quickly and delivers a complete strain field in the measuring area. In Fig. 3, the direct comparison of an ESPI measurement with classical strain gauges is shown. A composite plate with elliptical hole is loaded with a tensile force. The resulting strains in force direction have been measured with a set of 4 strain gauges along the axis of the hole. On the opposite side of the hole, the displacements were measured with ESPI and from there, the strains were calculated. The deviations of the local strain distribution along the axis of the hole between the ESPI measurement and strain gauge measurement are resulting from the averaging effect of the strain gauges. If the ESPI data are integrated in the same area as the dimension of the strain gauges, the results correlate excellently, as shown in Fig. 3. 


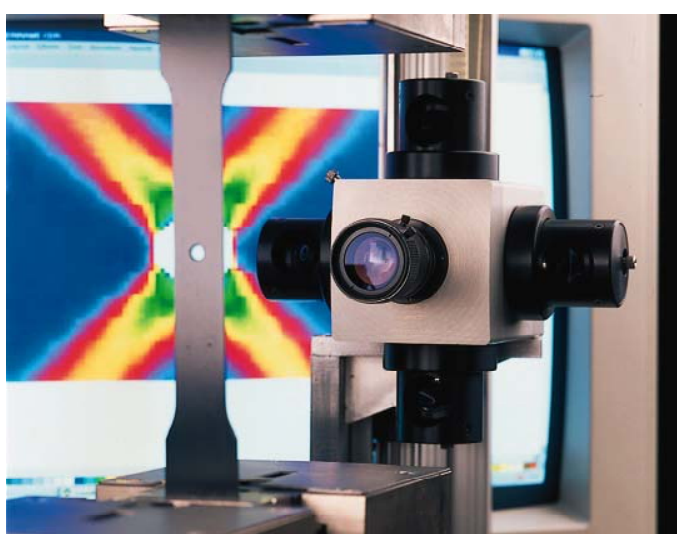

Fig. 2: 3D-ESPI System Q-300, /4/

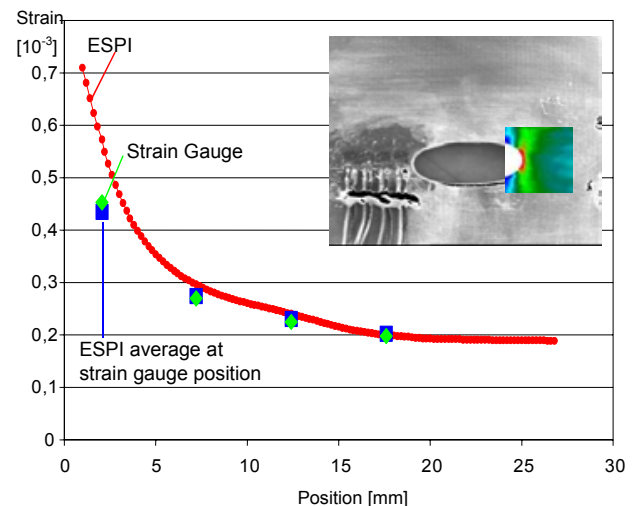

Fig. 3 : Comparison of ESPI measurement with strain gauges

High Strength Steel Weldings: The full field information of the ESPI measurement is especially valuable for investigations of non homogenous materials, such as e.g. joints. The optimisation of welding materials and processes requires detailed information about mismatches of the different properties of the base material, the heat effected zone and the welding itself, /5/. Fig. 4 shows a section of a specimen with a classical welding seam. The specimen was loaded in the x-direction up to a maximum and unloaded, afterwards. During the complete loading cycle, the strain field was measured in the observed area. Fig. 5 shows the resulting strain stress curves at different locations of the specimen. While the base material behaves linearly (blue curve), the point of maximum strain at the centre of the welding seam is clearly plastic with a large hysteresis effect (red curve). In comparison, the violet curve represents the integrated strains on the complete welding seam. A classical two-point strain measurement would show the average strain value according to the green curve.

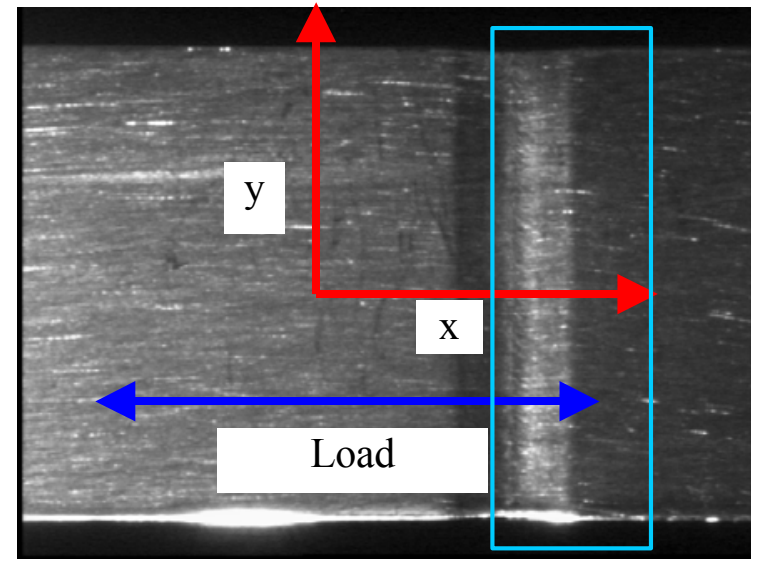

Fig. 4: Measuring field of a specimen with welding seam in tensile test

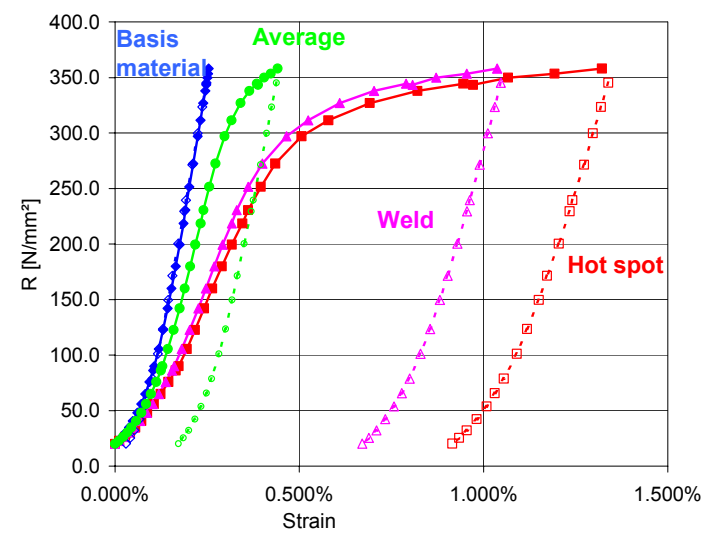

Fig. 5: Strain - stress curves at different positions of the specimen

Strain measurement on curved surfaces: As described above 3D-ESPI can determine the three components of deformation along with the strains of an object that are both given in Cartesian coordinates and these coordinates are denoted as an $\mathrm{O}_{\mathrm{xyz}}$ set of coordinates while on a flat surface. When the object under test has a curved surface, the Cartesian coordinates on the object are denoted as an $\mathrm{O}_{x^{\prime} y^{\prime} z^{\prime}}$ set of coordinates (usually the $\mathrm{z}$ axis is normal to object surface) and are usually different from the 3D-ESPI Cartesian coordinates as that from the flat surface, Fig. 6. Therefore, the components of deformation have to be transformed into the $\mathrm{O}_{x^{\prime} y^{\prime} z^{\prime}}$ coordinate system. Assuming that $\mathrm{u}, \mathrm{v}$ and $\mathrm{w}$ are the three components of deformation in the $\mathrm{O}_{\mathrm{xyz}}$ set of coordinates directly measured by 3D-ESPI they can directly be transformed into the component corresponding to the local coordinate system $\mathrm{O}_{x^{\prime} y^{\prime} z^{\prime}}$ by applying the transformation matrix $\mathbf{T}$ : 


$$
\left(\begin{array}{l}
u^{\prime} \\
v^{\prime} \\
w^{\prime}
\end{array}\right)=\overline{\bar{T}}\left(\begin{array}{l}
u \\
v \\
w
\end{array}\right)
$$

In order to determine the transformation matrix, a shape measurement of the object on the measured area is required. After the shape has been measured, the three components of deformation on the curved surface can be determined. The strains on a curved surface can be calculated according to the equations below.

$$
\varepsilon_{x}{ }^{\prime}=\frac{\partial u^{\prime}}{\partial x^{\prime}}, \quad \varepsilon_{x}{ }^{\prime}=\frac{\partial v^{\prime}}{\partial y^{\prime}}, \quad \gamma_{x y}{ }^{\prime}=\frac{\partial u^{\prime}}{\partial y^{\prime}}+\frac{\partial v^{\prime}}{\partial x^{\prime}}
$$

The shape of the curved surface can be measured with the same ESPI techniques as above, but using the inverse measuring principle: While the measuring object does not move, the illumination source of the ESPI system is laterally shifted. The resulting fringes correlate with the contour of the object and the optical set-up, /6/. With known geometry of the optical set-up and defined shift of the illumination the shape can be determined. For practical applications, the shape measuring function has been integrated into a miniaturized 3D ESPI system, Fig. 7.

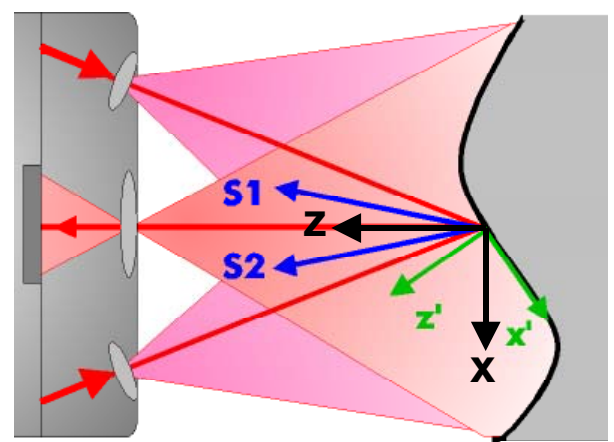

Fig. 6: Local coordinate system for 3D -ESPI measurement

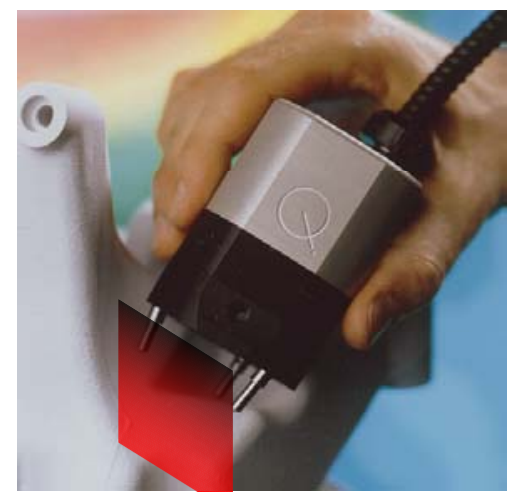

Fig. 7: Miniaturized 3D-ESPI system Q-100 for strain analysis on components, /4/

The ESPI sensor head can directly be attached to the surface of the measuring component with 3 feet allowing the inspection of an area of approx. $25 \times 35 \mathrm{~mm}^{2}$. As the test engineer generally knows about the critical areas, this measuring field is large enough to cover these areas and to record the interesting strain gradients. On the other hand it allowed the miniaturization of the system for easy application. The fixation of the sensor to the object compensates all rigid body movement and translation of the object due to the applied force and the system records only the local displacements and strains.

Application of Strain Measurement: The miniaturized 3D-ESPI system can be used on any type of engineering component. The high displacement measuring resolution of allows the determination of strains with a resolution in the range of 10 to $20 \mu \mathrm{m} / \mathrm{m}$. Therefore, the system is preferably being applied to fatigue investigations and stress analysis of safety relevant or other critical components in automotive, aerospace, etc.

Fig. 8 shows the application to an engine holder from cast aluminum in bending load. The sensor head is fixed into measuring position with a composite glue. After measurement of the shape in the measuring area, the load is applied and the are displacements recorded, Fig. 9. If the load is stepwise increased, the system can follow complete load cycles. With the knowledge of contour and displacement, the strain distribution on the surface can be calculated. 


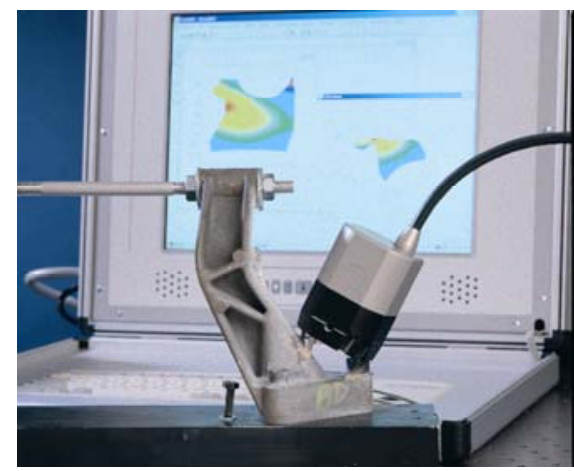

Fig. 8: Strain measurement on an engine holder

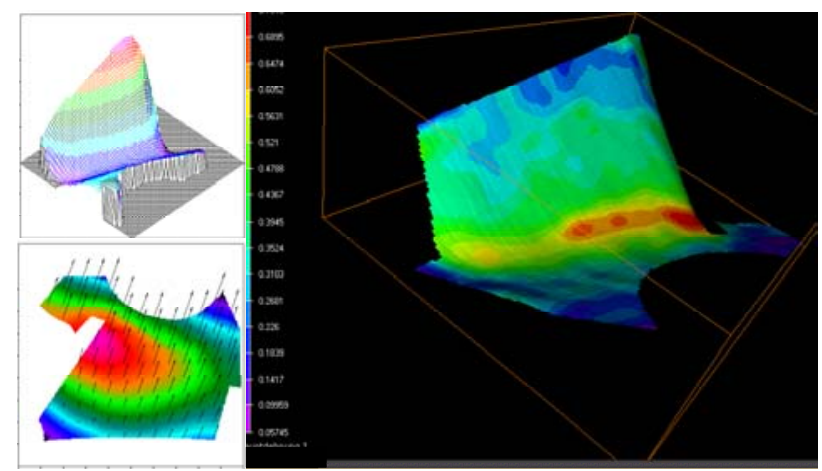

Fig. 9: Shape (top left), displacement (bottom left) principle strains (right) of the engine clamp

Combination with Finite Element Simulation: As the ESPI data carry the same information as Finite Element Analysis (FEA) an interface was developed to close the gap between the experiment and the numerical simulation. The ESPI data can be exported into a Virtual Reality Modelling Language (VRML) format, which can be imported by most CAE software packages. As example, Fig. 10 shows a test structure of a back plane for a convertible car, $/ 7 /$. The fixation points are critical areas with respect to the strength of the structure and were measured with the 3D-ESPI sensor. At each measuring position, the absolute coordinates of 4 measuring points were determined, Fig. 10. This allows the export of the calculated strain data directly into the CAD model for correlation with the FEA data, Fig. 11.
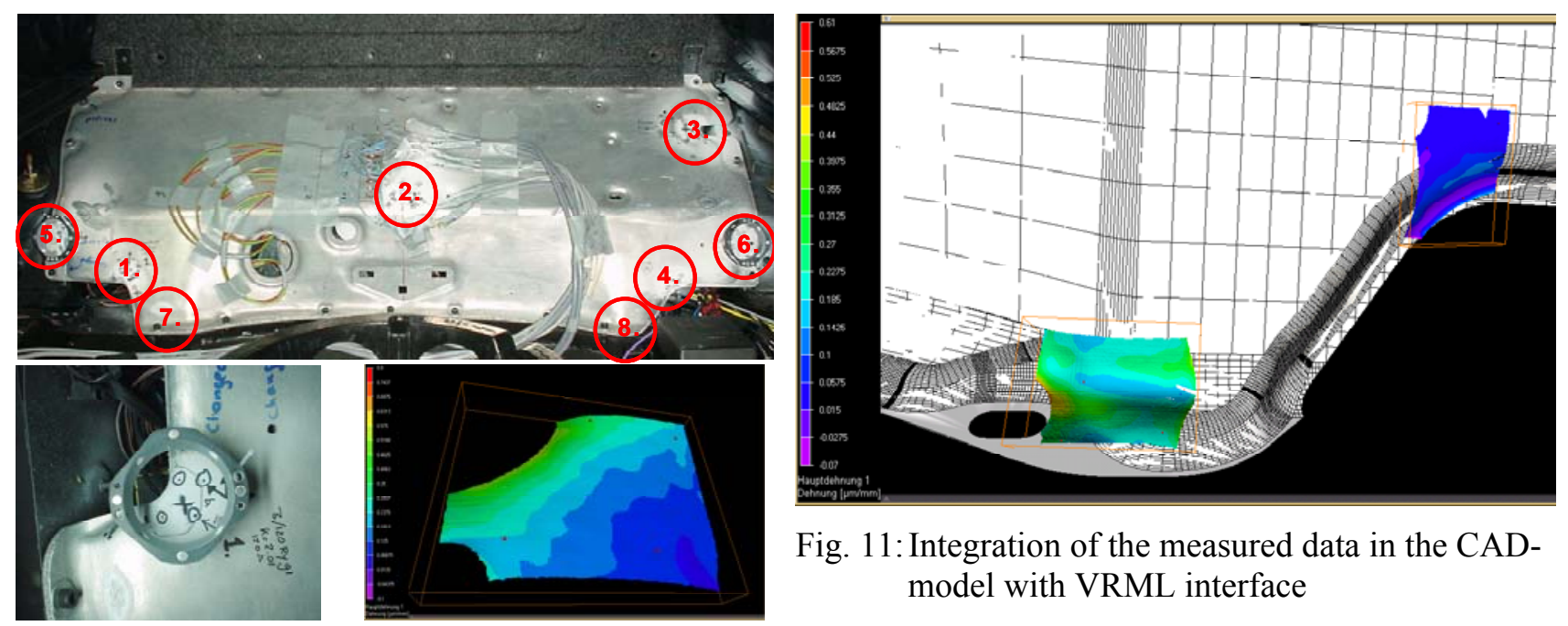

Fig. 11: Integration of the measured data in the CADmodel with VRML interface

Fig. 10:A1-foam test structure with measuring points (top), measuring position and strain (bottom)

Digital Image Correlation: While the ESPI technique gives excellent results in case of small deformation and strains, there is an increasing demand to understand the ductile behaviour of material. In case of several $10 \%$ strains respectively several millimetres displacement, the ESPI technique would require the handling of huge amounts of data. For such applications, a new technique has been developed, based on digital image correlation. This technique uses high resolution cameras to observe the surface of the measuring object, $/ 8 /$.

The principle is shown in Fig. 12. If a structure is observed with two cameras, each point is imaged on a certain pixel in the image plane of each camera. Knowing the exact positions of the camera with respect to each others, the position of the structure point can be identified in the coordinate system. For Digital 3D Image Correlation a stochastic pattern is applied to the surface of the object and observed with two cameras. Special correlation algorithms identify the stochastic patterns in both image planes with sub pixel accuracy. From there the position of each object point, which is observed by both cameras can be calculated, exactly. In this way, the 3D shape and position of the object is determined. 


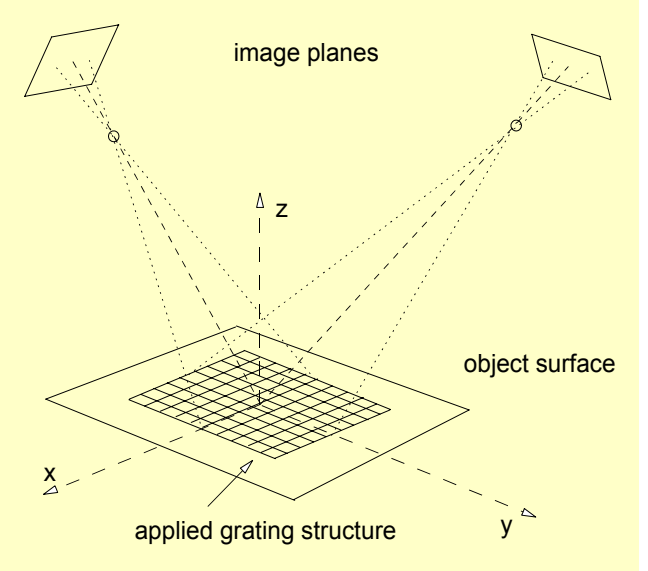

Fig. 12: Imaging of a structure in space with 2 cameras

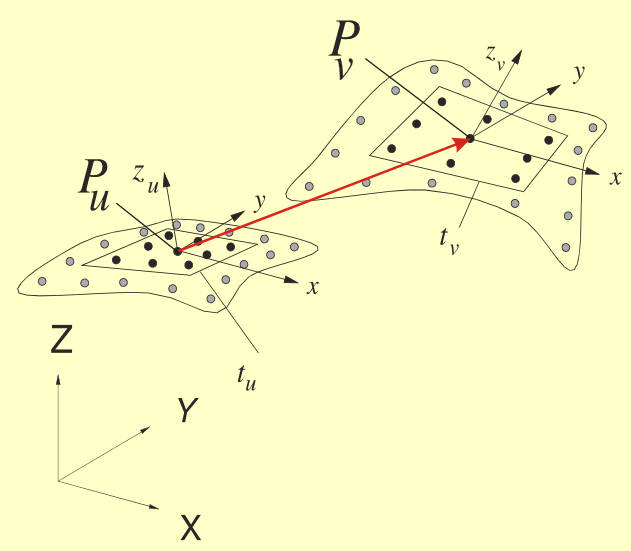

Fig. 13: Following the 3D displacement with digital image correlation

While the object is deformed, images can be recorded by both cameras and the same correlation procedures determine the three-dimensional shift of the object points, Fig. 13. As 3D image correlation delivers the shape and the three-dimensional displacement of each object point, the strains can be calculated, using the same formula above. Modern correlation algorithms achieve a resolution of displacement up to 1/100 pixels, resulting in strain levels of ca. $10^{-3}$. This is approx. a factor 10 to 50 less sensitive than ESPI technique, but on the other hand allows even the measurement of very large strain levels.

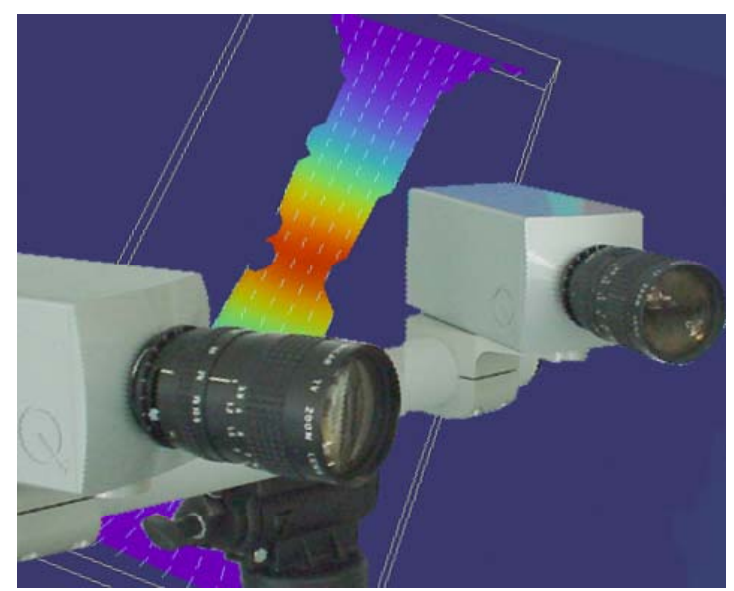

Fig. 14: Digital 3D image correlation system Q400
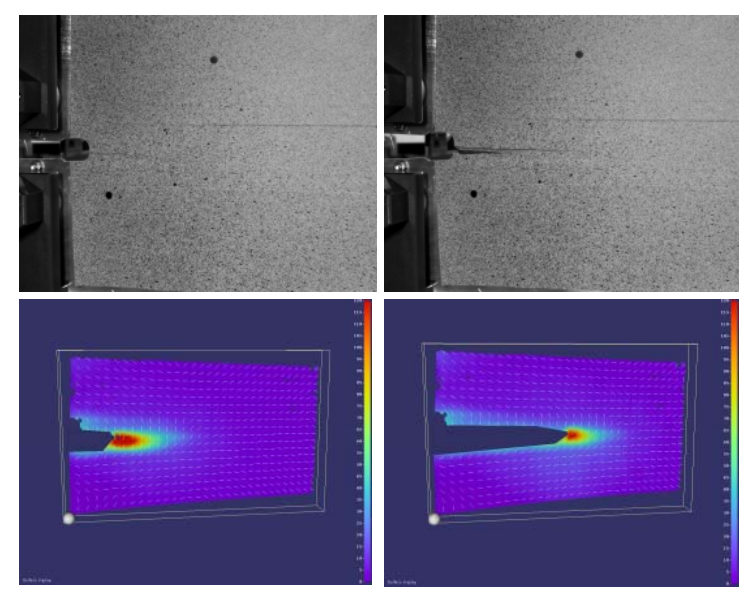

Fig. 15: Fracture test on large scale specimen

Measurement of Material Parameters: Fig. 14 shows a commercial 3D image correlation system. It consists of two high resolution cameras and a PC with software. In preparation of the measurement, the measuring object is covered with a stochastic pattern, which can be quickly applied by using white and black spray. The technique is especially useful for fracture mechanics applications. For investigations on large light weight structures fracture tests were carried out on Al-panels, Fig. 15. The field of view is approx. $500 \mathrm{~mm}$ x $500 \mathrm{~mm}$ and the force was applied in the image plane in vertical direction. The images show the development of the strain level while the crack is opening.

The great flexibility of the system also allows investigations on very small components. As an example, Fig. 16 shows the application on a tensile test of miniature Al-specimen to determine the material behaviour at high loads. The strains are recorded on all surface points, the position of maximum strain can be detected and evaluated. This is an important function for determination of e.g. forming limit diagrams and other parameters to characterize the forging features of materials. 


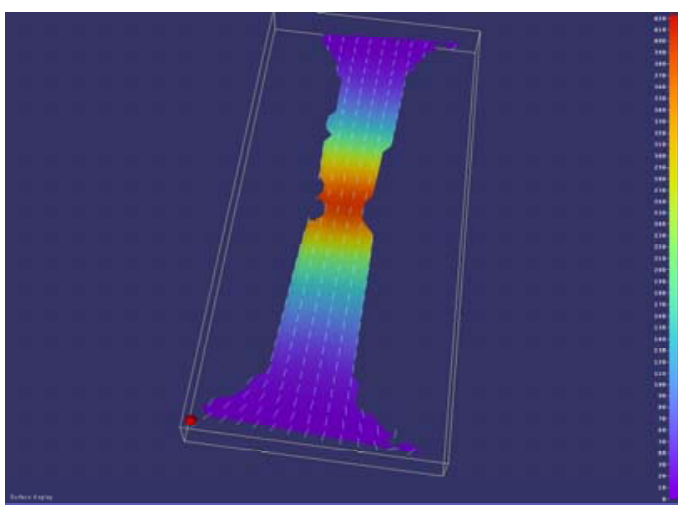

Fig. 16: Principal strain on a Al-specimen in tensile test

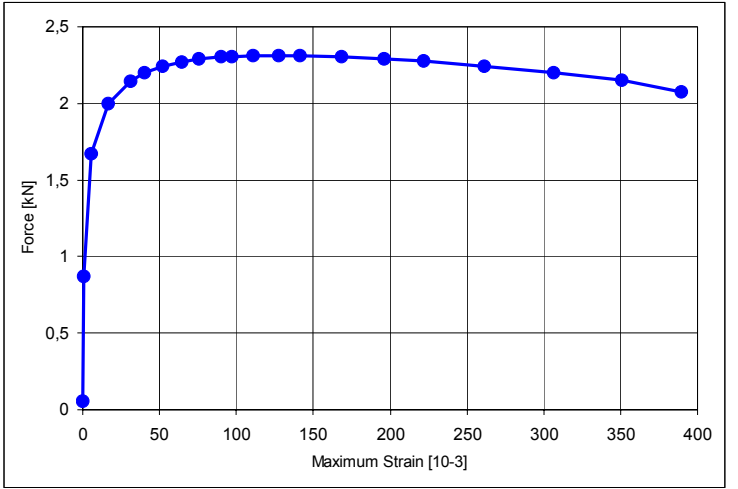

Fig. 17: Strain curve at position of maximum strain

Comparison: 3D-ESPI technique and Digital 3D Image Correlation Technique are both full field measuring techniques for three-dimensional displacement and strain/stress analysis for material and components. The range of applications is mainly determined by the expected measuring range and required accuracy. An indication about the practical measuring ranges in relation to typical object sizes is given in Fig. 18 for displacement and in Fig. 19 for strain measurement.

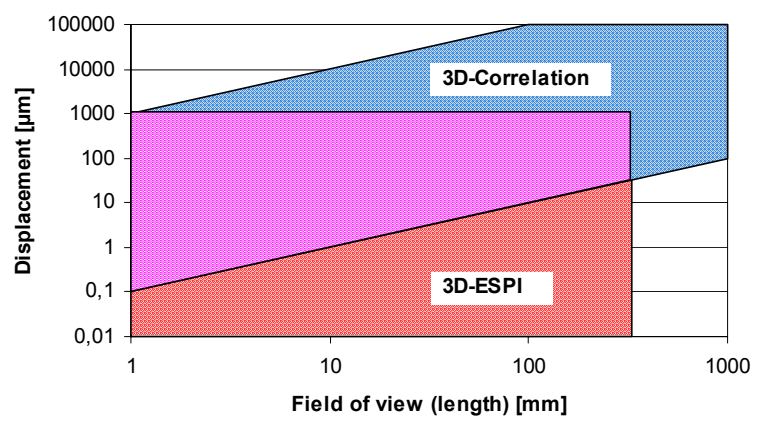

Fig. 18: Typical displacement measuring range for Fig. 19: Typical strain measuring range for 3D-ESPI and 3D Correlation techniques

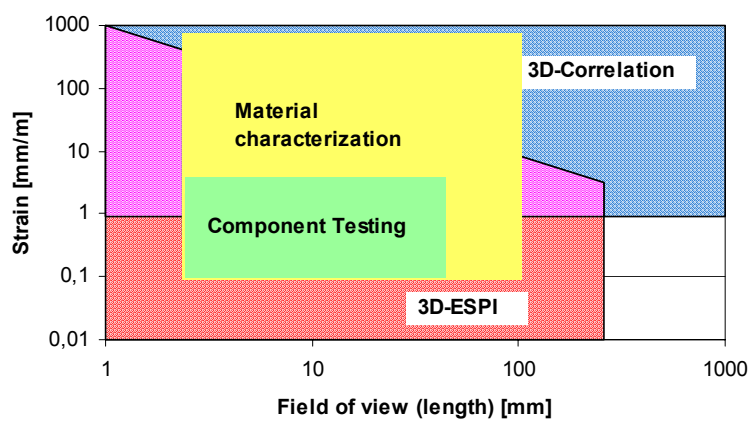

3D-ESPI and 3D Correlation techniques

Generally, for applications, which require strain/stress investigations also in the elastic-plastic material range, the higher sensitivity of the ESPI technique offers the best solution. Correlation techniques are preferred for measurements in the plastic range, for large measuring fields or for high measuring speeds. Table 1 summarizes some typical features of both techniques.

\begin{tabular}{|c|c|c|}
\hline & 3D-ESPI & Correlation \\
\hline Measuring area & \begin{tabular}{|ll}
$25 \times 35 \mathrm{~mm}^{2}$ & with Q-100 \\
$1 \times 1.5 \mathrm{~mm}^{2} \ldots$ & $200 \times 300 \mathrm{~mm}^{2}$ \\
& with Q-300
\end{tabular} & $\begin{array}{c}1 \times 1 \mathrm{~mm}^{2} \ldots 2 \times 2 \mathrm{~m}^{2} \\
\text { with Microscope optics } \\
\text { even smaller }\end{array}$ \\
\hline $\begin{array}{c}\text { Measuring sensitivity } \\
\text { Displacement } \\
\text { Strain }\end{array}$ & $\begin{array}{l}0.03 \ldots 0.1 \mu \mathrm{m} \\
10 \ldots 20 \mu \mathrm{m} / \mathrm{m}\end{array}$ & $\begin{array}{l}1 / 10 \ldots 1 / 100 \text { pixel, typ. } 10 \mu \mathrm{m} \\
500 \ldots 1000 \mu \mathrm{m} / \mathrm{m}\end{array}$ \\
\hline $\begin{array}{l}\text { Measuring speed } \\
\text { (image acquisition) }\end{array}$ & Static $(1 \ldots 2 \mathrm{~s} / \mathrm{step})$ & $\begin{array}{l}\text { Static and dynamic } \\
\text { Camera acquisition rate : } \\
16 \mathrm{~Hz} \text { (standard) }\end{array}$ \\
\hline
\end{tabular}




\begin{tabular}{|l|l|l|}
\hline & & \multicolumn{1}{|c|}{$>\mathrm{kHz}$ (High Speed) } \\
\hline Object geometry & Any & Any \\
\hline Surface quality & $\begin{array}{l}\text { Not shiny, } \\
\text { No marking }\end{array}$ & Stochastic pattern required \\
\hline
\end{tabular}

Table 1: Comparison of 3D ESPI and Digital 3D Correlation Techniques

Summary: Full field optical measuring techniques allow fast, three-dimensional strain/stress analysis on specimen and components. For high resolution measurement and for strain/stress analysis on components, the ESPI has been proven its ability as problem solving tool in research and in industry. The possibility to correlate the measuring results with Finite Element Analysis enables more efficient and faster development of components. The flexible Digital 3D Image Correlation Technique enlarges the measuring range of optical full field strain/stress analysis techniques to large deformations and strains.

\section{References:}

/1/ R. Jones, C. Wykes, "Holographic and Speckle Interferometry," Cambridge University Press (1989).

/2/ K. Creath, "Phase-shifting speckle interferometry," Appl. Opt. 24 (18), 3053 -3058, (1985).

/3/ L. Yang, A. Ettemeyer: Strain measurement by three-dimensional electronic speckle pattern interferometry: potentials, limitations, and applications, Optical Engineering, SPIE, May 2003, Vol. 42, No. 5

/4/ www.dantec-ettemeyer.com, homepage and product information of Dantec Ettemeyer GmbH, Ulm, Germany

/5/ R. Schubach, J. Hammer, A. Ettemeyer: Investigations on laser welded high strength steel with speckle interferometry, SEM Spring Conference, Milwaukee, USA, 10-12. June 2002

/6/ C. Joenathan, B. Pfister, H.J. Tiziani: Contouring by electronic speckle pattern interferometry employing dual beam illumination, App. Opt. 29 (13), 1905-1911 (1990)

/7/ T. Siebert, K. Splitthof, A. Ettemeyer: A Practical Approach to Solve the Problem of the Absolute Phase in Speckle Interferometry, Journal for Holography and Speckle, Vol. 1, No. 1 (2004)

/8/ C. Herbst, K. Splitthof, A. Ettemeyer: New Features in Digital Image Correlation Techniques, CMOI conference, 4th French Colloquium "Méthodes et Techniques Optiques pour 1'Industrie", Belfort, France, 17.-21.11.2003 